\title{
Planck-Scale Gravity Test at PETRA
}

\author{
Vahagn Gharibyan, Klaus Balewski \\ Deutsches Elektronen-Synchrotron DESY, Hamburg, Germany \\ Email: Vahagn.Gharibyan@desy.de, Klaus.Balewski@desy.de
}

Received 6 March 2016; accepted 24 May 2016; published 27 May 2016

Copyright (C) 2016 by authors and Scientific Research Publishing Inc.

This work is licensed under the Creative Commons Attribution International License (CC BY). http://creativecommons.org/licenses/by/4.0/

(c) (i) Open Access

\begin{abstract}
Quantum or torsion gravity models predict unusual properties of space-time at very short distances. In particular, near the Planck length, around $10^{-35} \mathrm{~m}$, empty space may behave as a crystal, singly or doubly refractive. This hypothesis, however, remains uncheckable for any direct measurement since the smallest distance accessible in experiment is about $10^{-19} \mathrm{~m}$ at the LHC. Here we propose a laboratory test to measure space birefringence or refractivity induced by gravity. A sensitivity $10^{-31} \mathrm{~m}$ for doubly and $10^{-28} \mathrm{~m}$ for singly refractive vacuum could be reached with PETRA $6 \mathrm{GeV}$ beam exploring UV laser Compton scattering.
\end{abstract}

\section{Keywords}

\section{Experimental Test of Vacuum, Planck Energy, Gravitation, Laser Compton Scattering, Charged} Particle Beams in Accelerators

\section{Introduction}

The quantum formalism cannot be directly applied to gravitation and that is one of the major problems on a way of understanding and describing the physical reality. An important reason for this is the dynamical space concept adopted in general relativity, the currently accepted theory of gravity which states that any mass or particle modifies the space geometry (or metrics). On the other hand, the successful quantum theories within the Standard Model operate only in a fixed geometry space. For instance, observed violations of the discrete symmetries such as space, charge and time parities are attributed to the particles and their interactions while the scene of the interactions, the space-time, is considered to remain perfectly symmetric [1]. These two faces of space are believed to unify at distances near the Planck length $l_{P}=1.6 \times 10^{-35} \mathrm{~m}$ (or mass $M_{P}=1.2 \times 10^{19} \mathrm{GeV}$, natural units are assumed throughout the letter). At this scale gravity is expected to be similar in strength to the electroweak and strong forces and quantum effects become important for the gravitational field. String theory and loop quantum gravity theory are prominent candidates which set a framework to make predictions in that energy domain. In many cases, unconventional space-time properties are suggested, such as vacuum refractivity [2] and/or 
birefringence [3].

Such effects may be studied by using lasers and high energy accelerator beams as recommended in Ref. [4]. The proposed experiment at PETRA will probe the vacuum symmetry in a search for a handedness or chirality of the empty space presumed by quantum gravity. A figure of merit is circular birefringence $\Delta n=n_{L}-n_{R}$ of space, with $n_{L(R)}$ being the refraction index of left (right) helicity photons traversing the space. Average refraction $n=\left(n_{L}+n_{R}\right) / 2$ will be tested with additional instrumentation.

In the following, quantitative theoretical estimates and existing experimental limits are quoted, the formalism of the suggested method is presented, and the proposed experimental setup is described. Expected performance, experimental reach with statistical and systematic accuracy estimates are discussed as well.

\section{Photon Dispersion at Planck-Scale}

Since Planck mass $M_{P}=\sqrt{c \hbar / G}$ is build from the speed of light and fundamental Planck and gravitational constants, this mass scale is considered to be relativistic and quantum gravitational. Most general modification of photon dispersion relation at lowest order of Planck mass could be expressed as [5] [6]

$$
\omega^{2}=k^{2} \pm \xi \frac{k^{3}}{M_{P}}
$$

where $\omega$ and $k$ are photon's energy, momentum, respectively, while the $\xi$ is a dimensionless parameter and the \pm signs stand for opposite helicity photons. This is main relation we are going to test at PETRA.

Several theories are predicting or supporting the relation (1). The Planck scale quantum gravity modifies the Maxwell equations by adding extra terms proportional to the Planck length [7]:

$$
\begin{gathered}
\frac{\partial \boldsymbol{E}}{\partial t}=\nabla \times \boldsymbol{B}-2 \xi l_{P} \nabla^{2} \boldsymbol{B} \\
\frac{\partial \boldsymbol{B}}{\partial t}=-\nabla \times \boldsymbol{E}-2 \xi l_{P} \nabla^{2} \boldsymbol{E}
\end{gathered}
$$

which leads to a deformed energy-momentum or dispersion relation (1). In the above equations, $\boldsymbol{E}$ and $\boldsymbol{B}$ describe the electromagnetic field. More general expressions accounting for space anisotropy are derived in Ref. [8]. Using conventional definition $n=\mathrm{d} \omega / \mathrm{d} k$, it is easy to verify that Equations (1)-(3) introduce a chiral vacuum with an energy dependent birefringence

$$
\Delta n=3 \times 10^{-19} \cdot \xi \cdot \omega[\mathrm{GeV}]
$$

where the magnitude of $\xi$ defines the characteristic energies or distances where quantum-gravity effects become sizeable. In the simplest possible picture, this only happens at the Planck scale, and hence $\xi=1$. However, the running of fundamental constants with energy may require quantum gravity to become active a few orders of magnitude below the Planck scale. The parameter $\xi$ is there to account for such effects.

Another possible source of vacuum chirality is described by torsion gravity, an extension of the general relativity into the microscopic world to include particles' spins-for a review see [9]. In general, the spin gravity (space torsion) is considered to be weaker than the mass gravity (space curvature). However, near the Planck scale it may become detectable. Following Ref. [10], from the electromagnetic field Lagrangian

$$
\mathcal{L}=-\frac{1}{4} F_{\mu \nu} F^{\mu \nu}+q T^{\mu \nu \rho}\left(\partial^{\sigma} F_{\mu \nu}\right) F_{\rho \sigma}
$$

with a torsion tensor $T^{\mu v \rho}$ and free parameter $q$ one derives a dispersion relation quite similar to Equation (1)

$$
\omega^{2}=k^{2} \pm q S_{0} k^{3}
$$

where $S_{0}$ stands for a time component of the contorsion vector.

Myers and Pospelov [11] derived the expression (1) within effective field theory with dimension 5 operators. A similar effect is calculated in Ref. [12] exploring graviton interaction with electromagnetic field in one-loop approximation. In our setup the gravitons emerge from the gravitational field of Earth. In summary, chiral space is a universal feature of Planck-scale gravity, in the sense that it is predicted by a large diversity of theories.

A nonbirefringent gravitational space is also possible and has been predicted within String theories using 
D-brane formalism. In Ref. [13] a polarization independent refractivity

$$
n-1=\zeta \frac{k}{M_{P}}
$$

is obtained for the space-time foam near the Planck length. Here we use $\zeta$ instead of the $\xi$ to distinguish between the nonchiral and chiral space. In principle, both types may occur in the same vacuum at different scales $\zeta$ and $\xi$. Both gravity induced effects, namely birefringence and refractivity, share the common feature that their strength is growing with the photon energy. This is in contrast to the usual condensed matter or electromagnetic, nontrivial vacua where the refraction effects are suppressed by powers of the energy [14] [15]. Such growth should allow to approach Planck scale at the PETRA as will be shown below.

\section{Current Limits}

Experimental limits on space chirality are set by astrophysical observations exploring birefringence induced depolarization of the linear light which comes from distant cosmological sources [16]. The limits, however, are based on assumptions about the origin, spatial or temporal distribution of the initial photons, and their possible interactions during the travel. Another critical assumption is a uniformly distributed birefringence over cosmological distances. The most stringent limit $\xi<2.4 \times 10^{-15}$ is set by Ref. [17] based on photons with polarization $0.63 \pm 0.30$ in an energy range from 100 to $350 \mathrm{keV}$ from GRB041219a [18]. Sensitive particle-physics effects have been suggested to test quantum gravity, mainly using threshold energies [19]. Applying cosmic ray constraints on photon decay and vacuum Cherenkov radiation [20], one arrives to $\zeta<30$ and $\zeta<300$ limits, respectively.

For the space refractivity, there are astrophysical observations interpreted [21] as $\zeta \sim 10$. This is derived from energy dependent time delay measurements of photons from distant sources. Similar to the results derived from polarized photons of cosmological origin, strong assumptions have to be made on the source of these photons. In addition, quoted astrophysical constraints are valid only for photon-virtual graviton loop interactions, since the photon path is essentially free from gravitational fields.

PETRA measurements could shed light on the quantum-gravity promoted space chirality and refractivity including effects introduced by Earth gravitons. In the laboratory the Planck scale can be accessed by exploring the extreme sensitivity of the high energy Compton scattering to the vacuum refraction as discussed in the following.

\section{Compton Scattering Affected by Gravity}

Let us denote by $\omega_{0}, \omega, \theta_{0}, \theta$ the energies and angles of the incident and scattered photons relative to the initial electron direction as illustrated in Figure 1. Then, according to Ref. [20], for the high energy Compton scattering in a vacuum with $n \approx 1$ (up to $\mathcal{O}\left((n-1)^{2}\right)$ terms), the energy-momentum conservation yields

$$
n-1=\frac{\mathcal{E}}{2 \gamma^{2}(\mathcal{E}-\omega)}\left(1+x+\theta^{2} \gamma^{2}-x \frac{\mathcal{E}}{\omega}\right)
$$

where $\gamma, \mathcal{E}$ are the Lorentz factor and energy of the initial electron, $x \equiv 4 \gamma \omega_{0} \sin ^{2}\left(\theta_{0} / 2\right) / m$, and $n$ is the refraction index for the direction $\theta$ and energy $\omega$. This formula is more general than Equation (3) of Ref. [20]. The difference is in a factor $E /(E-\omega)$, because in contrast to [20] the final photon mass squared $k_{\mu}^{2}=\omega^{2}\left(1-n^{2}\right)$ is not neglected for this Letter.

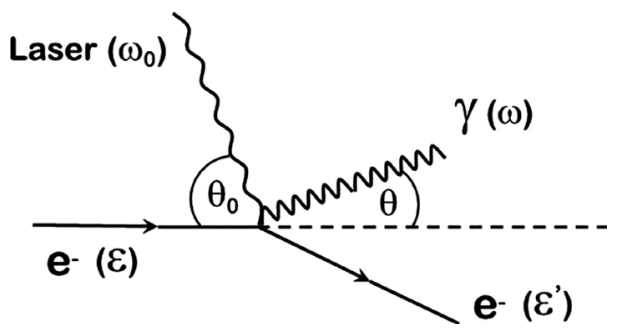

Figure 1. Compton scattering schematics. 
Substituting $n-1$ in the Equation (8) by the gravitational refractivity from the Equation (7) we can estimate how the quantum gravity would change the scattered photons' maximal energy $\omega_{m}$ (Compton edge, at $\theta=0$ ). The expected shift of the Compton edge is

$$
\Delta \omega_{m} \equiv \omega_{m}(n)-\omega_{m}(1)=\frac{32 \gamma^{6} \omega_{0}^{2} \sin ^{4}\left(\theta_{0} / 2\right)}{(1+x)^{4}} \frac{\zeta}{M_{P}}
$$

relative to the vacuum $(n=1)$ kinematics. For optical lasers and head-on collision the kinematic factor $x \approx 2 \times 10^{-5} \gamma$ and the right-hand side of Equation (9) grows as $\gamma^{6}$ at $\mathrm{GeV}$ energies slowing down to $\gamma^{2}$ growth above $\mathrm{TeV}$ energies. At sufficiently high $\gamma$, the huge value of $M_{P}$ is compensated and the energy shift becomes detectable. Hence, this effect allows quantum-gravity induced space refractivity to be measured at PETRA by laser Compton scattering off electrons with $\gamma=11742$.

In order to probe space birefringence, one needs to measure the refractivity in Equation (8) for scattered photons of opposite helicity. This may be achieved by exploring circularly polarized initial laser beams and helicity conservation. The polarization of the secondary photons in the case of scattering on unpolarized electrons is shown in Figure 2, using formulas from Ref. [22] [23]. At $\omega=\omega_{m}$ the polarization transfer is complete, such that the helicity of the Compton edge photons is fully defined by the laser light helicity. Consequently, in a birefringent vacuum the Compton edge energy is laser helicity dependent. Evaluating Equation (8) for left and right helicity photons at $\theta=0$ yields

$$
\Delta n=n_{L}\left(\omega_{m}^{L}\right)-n_{R}\left(\omega_{m}^{R}\right)=\frac{(1+x)^{2}}{\gamma^{2}} A
$$

where $\omega_{m}^{L}$ and $\omega_{m}^{R}$ are the highest energies for the Compton opposite helicity photons and $A=\left(\omega_{m}^{L}-\omega_{m}^{R}\right) /\left(\omega_{m}^{L}+\omega_{m}^{R}\right)$ is an energy asymmetry.

Combining Equation (10) with the gravitational birefringence from the Equation (4) we arrive to

$$
A=\frac{8 \gamma^{4} \omega_{0} \sin ^{2}\left(\theta_{0} / 2\right)}{(1+x)^{3}} \frac{\xi}{M_{P}}
$$

which proves that for PETRA values of $\gamma$ the Planck scale space birefringence generates a measurable asymmetry.

In contrast to the astrophysical methods, an accelerator Compton experiment is sensitive to the local

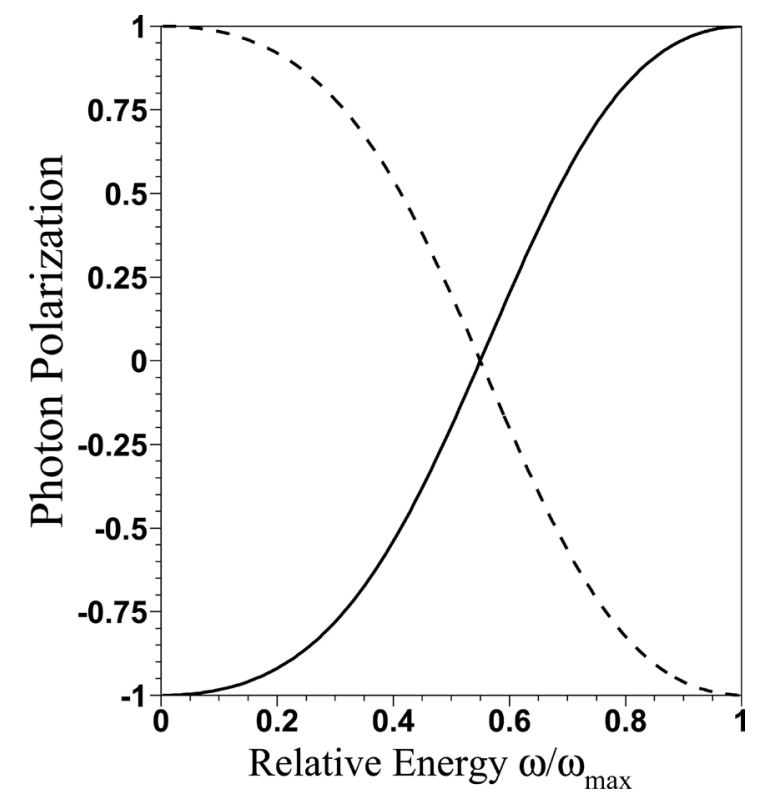

Figure 2. Polarization of the Compton scattered photon on a $6 \mathrm{GeV}$ electron as a function of the photon energy. The solid and dotted lines correspond to the initial laser light helicity: +1 solid, -1 dotted. 
properties of space at the laser-electron interaction point and along the scattered photon direction. Hence, space isotropy tests are also possible as the accelerator rotates together with Earth. For any preferred direction the measured birefringence is expected to change as the scattered photon beam sweeps a circle over the celestial sphere. For a given direction $(\delta, \alpha)$ of the photon beam and a possible anisotropy axis $\left(\delta_{0}, \alpha_{0}\right)$ one expects

$$
\Delta n=\Delta n_{0}\left(\cos \delta \cos \delta_{0} \cos \left(\alpha-\alpha_{0}\right)+\sin \delta \sin \delta_{0}\right)
$$

where $\Delta n_{0}$ is the maximal birefringence, along the declination $\delta_{0}$ and right ascension $\alpha_{0}$. Despite of tight limits set by low energy high precision experiments on space anisotropy [24] the accelerator isotropy test is a valuable and complementary test at high energies.

\section{Proposed Experiment}

In order to measure space birefringence and refractivity we propose a laser Compton experiment to be performed at the PETRA SW section. The experiment will bring into collision PETRA bunches and light pulses from a mode locked laser to produce Compton photons. Scattered photons and positrons, separated by a beam dipole magnet, are registered by downstream detectors. Positions and energies of the scattered secondaries will mainly be measured in single particle detection mode. Positron beam position is measured using PETRA high precision BPM (Beam Position Monitor) system [25]. Laser beam intensity and polarisation will be monitored in a light Analyzer Box. A schematic arrangement of the proposed experiment is presented in Figure 3. Measured positions $X_{e}$ at laser light opposite helicities will allow to derive space birefringence while the refractivity could be accessed using in addition horizontal positions of the beam $X_{B}$ and the Compton photon $X_{\gamma}$.

We plan to run the experiment in "parasitic" mode without disturbing user operations or affecting machine beam quality.

\subsection{Accelerator}

PETRA III [26] is a third generation light source with $6 \mathrm{GeV}$ high quality positron beam. Main operational parameters of the machine [27] are collected in Table 1.

Time resolved state with 40 or 60 bunches is the main working mode of the machine. Top-up running allows long-term stable operation with constant current. On Figure 4 a typical performance of the PETRA is shown over a 24 hours period.

At the planned laser-positron interaction point PETRA beam has a horizontal dispersion $D_{x}=0.139 \mathrm{~m}$ and following Twiss parameters

$$
\begin{gathered}
\alpha_{x}=0.427, \quad \beta_{x}=11.114 \mathrm{~m} \\
\alpha_{y}=-1.311, \quad \beta_{y}=19.945 \mathrm{~m}
\end{gathered}
$$

with RMS beam sizes $\sigma_{x}=106 \mu \mathrm{m}$ and $\sigma_{y}=24 \mu \mathrm{m}$ and RMS divergences $\sigma_{x^{\prime}}=10 \mu \mathrm{rad}$ and $\sigma_{y^{\prime}}=2 \mu \mathrm{rad}$.

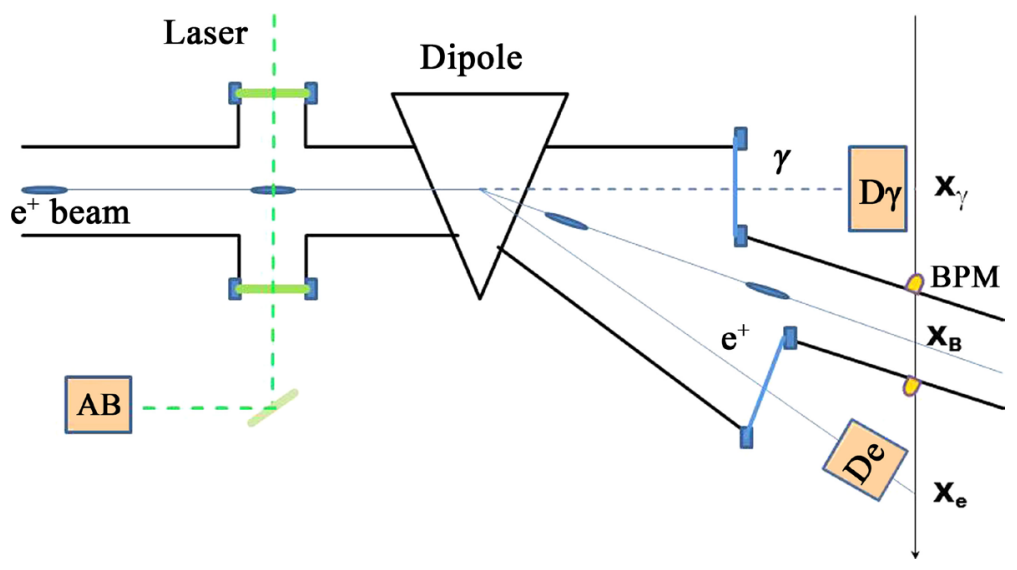

Figure 3. Schematic overview of the experiment. 
Table 1. PETRA III parameters.

\begin{tabular}{ll}
\hline & $6.0 \mathrm{GeV}$ \\
Positron energy & $2304 \mathrm{~m}$ \\
Circumference of the storage ring & 3840 \\
Harmonic number (buckets) & $40-960$ \\
Number of bunches & $192 \mathrm{~ns}-8 \mathrm{~ns}$ \\
Bunch separation & $100 \mathrm{~mA}$ (top-up mode) \\
Positron beam current & $1 \mathrm{~nm} \cdot \mathrm{rad}$ (rms) \\
Horizontal emittance & $0.01 \mathrm{~nm} \cdot \mathrm{rad}$ (rms) \\
Vertical emittance & $0.1 \%$ (rms) \\
Positron beam energy spread & $499.564 \mathrm{MHz}$ \\
RF & $7.685 \mu \mathrm{sec}$ \\
Revolution time & $130.121 \mathrm{kHz}$ \\
Revolution frequency & $44 \mathrm{psec}$ \\
Bunch length (rms) & $1 \mathrm{MeV}$ \\
Positron energy loss per turn from dipoles & $6 \mathrm{MeV}$ \\
Overall positron energy loss per turn & $2 \mathrm{~h}$ \\
Positron beam lifetime (time resolved mode) & \\
\hline &
\end{tabular}

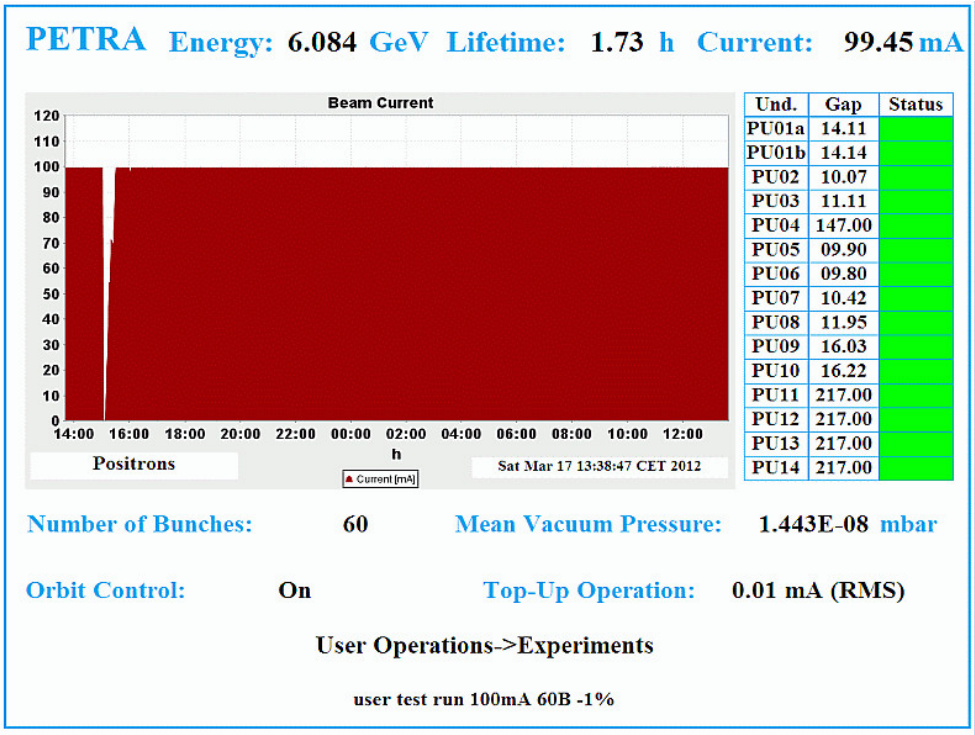

Figure 4. PETRA online status display [28].

\subsection{Laser}

Laser in this application should provide sufficient luminosity for high Compton rates at single or few particles operational mode. In addition the light wavelength should be possibly short to extract and detect the scattered positrons within available limited distance from interaction point. These two requirements somewhat contradict 
each other since the Compton cross-section is falling toward shorter wavelengths. Thus, at acceptable wavelengths the laser power demand is so high that appropriate CW lasers are not available in market. Since high power Q-switched lasers are not adequate for single particle mode applications, we have to choose among mode-locked lasers. An example of commercially available laser which meets our needs is Coherent Paladin UV laser [29] with parameters listed in Table 2.

The laser light will be delivered to the interaction chamber by a single mode, polarization maintaining fiber. This will provide fixed position of the light at the interaction point independent of the polarization state. At the end of the fiber a quarter wave plate will convert linear light into a circular one which then will be focused $<10 \mu \mathrm{m}$ to the positron beam. Polarization state of the interacting laser photons will be controlled by an electro-optical Pockels-cell device installed upstream of the fiber. Light polarization and intensity will be constantly monitored at laser beam dump, in Analyzer Box.

\subsection{Experimental Sensitivity}

Given the accelerator energy of $6 \mathrm{GeV}$, laser wavelength of $355 \mathrm{~nm}$ and $e \gamma$ crossing angle of $90^{\circ}$, gravity induced effects could be calculated using Equation (9) and Equation (11). Single refraction in crystal space will shift the Compton edge while laser helicity flip will produce an energy asymmetry induced by double refraction. Magnitude of these effects are shown in Figure 5. Experimental reach of the experiment is then defined by accuracies for the energy and asymmetry measurements as well as limiting systematic effects. We expect precisions of $\Delta \omega_{m} / \omega_{m}=10^{-3}$ to $10^{-4}$ for energy and $10^{-7}$ to $10^{-8}$ for asymmetry measurements which are corresponding to upper right and lower left regions on Figure 5. Detailed calculations will be presented in the following sections.

\subsection{Beamline}

We plan to use existing beamline and interaction vacuum chamber build for PETRA-III Laser-Wire project [30] (see Figure 6). The chamber has horizontal and vertical optical entry-exit ports (windows) for the laser. For our application we will use vertical ports which assumes careful stress-less mounting of the laser windows to preserve circular polarization of the light.

A vacuum exit window (2 $\mathrm{mm} \mathrm{Al}$ ) for the Compton photons is located at $7.8 \mathrm{~m}$ distance from the interaction chamber. Beamline essential components are listed in Table 3 and are drawn in Figure 7.

Beam position monitors (BPM) near and around the interaction point are dedicated for beam position, slope

\begin{tabular}{cc} 
Table 2. Coherent Paladin laser specifications. \\
\\
Wavelength & $355 \mathrm{~nm}$ \\
Output power & $>8 \mathrm{~W}$ \\
Repetition rate & $80 \pm 1 \mathrm{MHz}$ \\
Pulse length & $>15 \mathrm{ps}$ at $1064 \mathrm{~nm}$ \\
Spatial mode & $\mathrm{TEM} 00$ \\
M2 & $<1.2$ \\
Beam diameter & $1 \% \pm 15 \% \mathrm{~mm}$ \\
Beam divergence & $<550 \mu \mathrm{rad}$ \\
Beam ellipticity & $0.9 \mathrm{to} 1.1$ \\
Pointing stability & $<20 \mu \mathrm{rad} /{ }^{\circ} \mathrm{C}$ \\
Polarization linear & $>100: 1, \mathrm{vertical}$ \\
Long-term power stability & $< \pm 2 \%$ \\
\hline
\end{tabular}




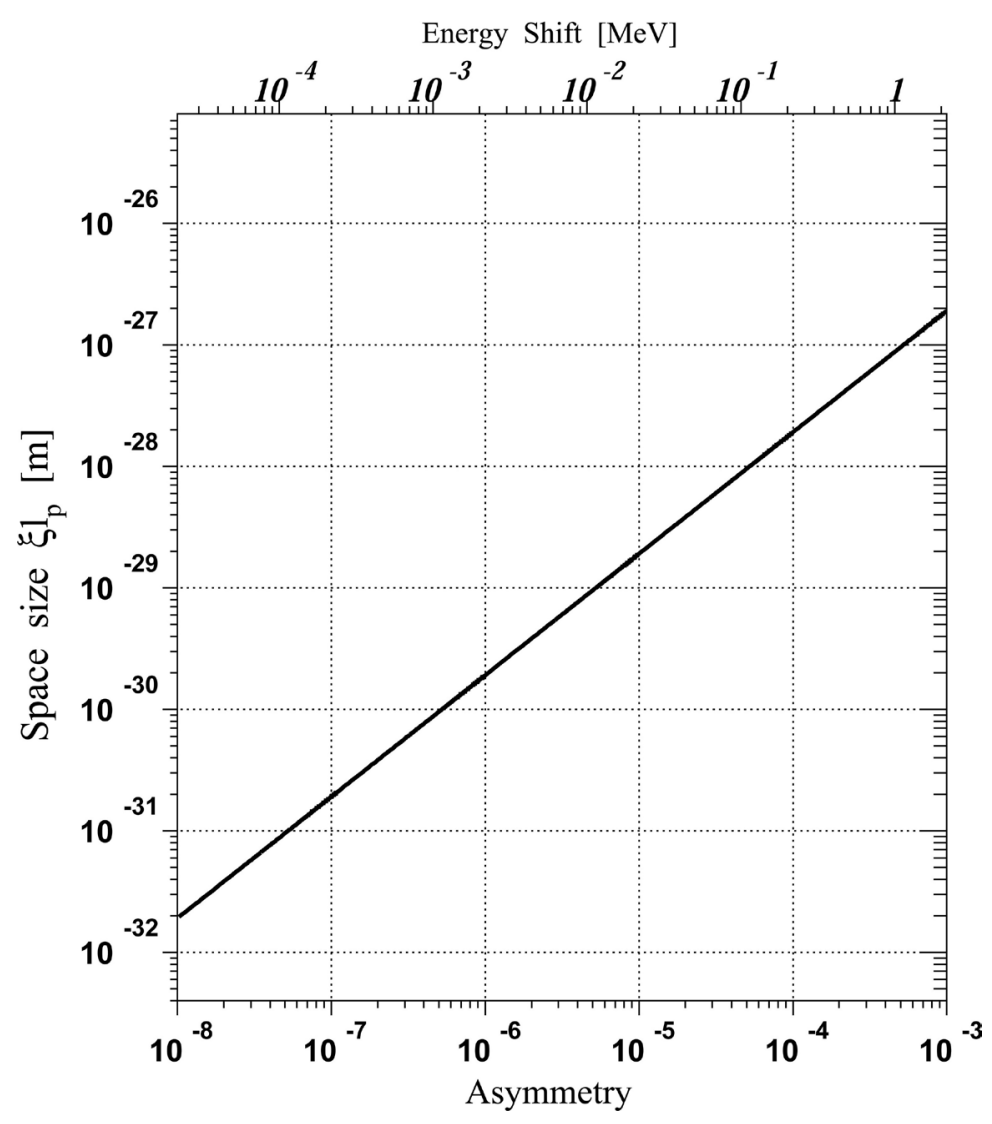

Figure 5. Sensitivity of the PETRA III for vacuum birefringence and refractivity. Birefringence at the scale $\xi$ will produce a Compton edge asymmetry (lower scale) while the refractivity produces absolute energy shifts (upper scale).
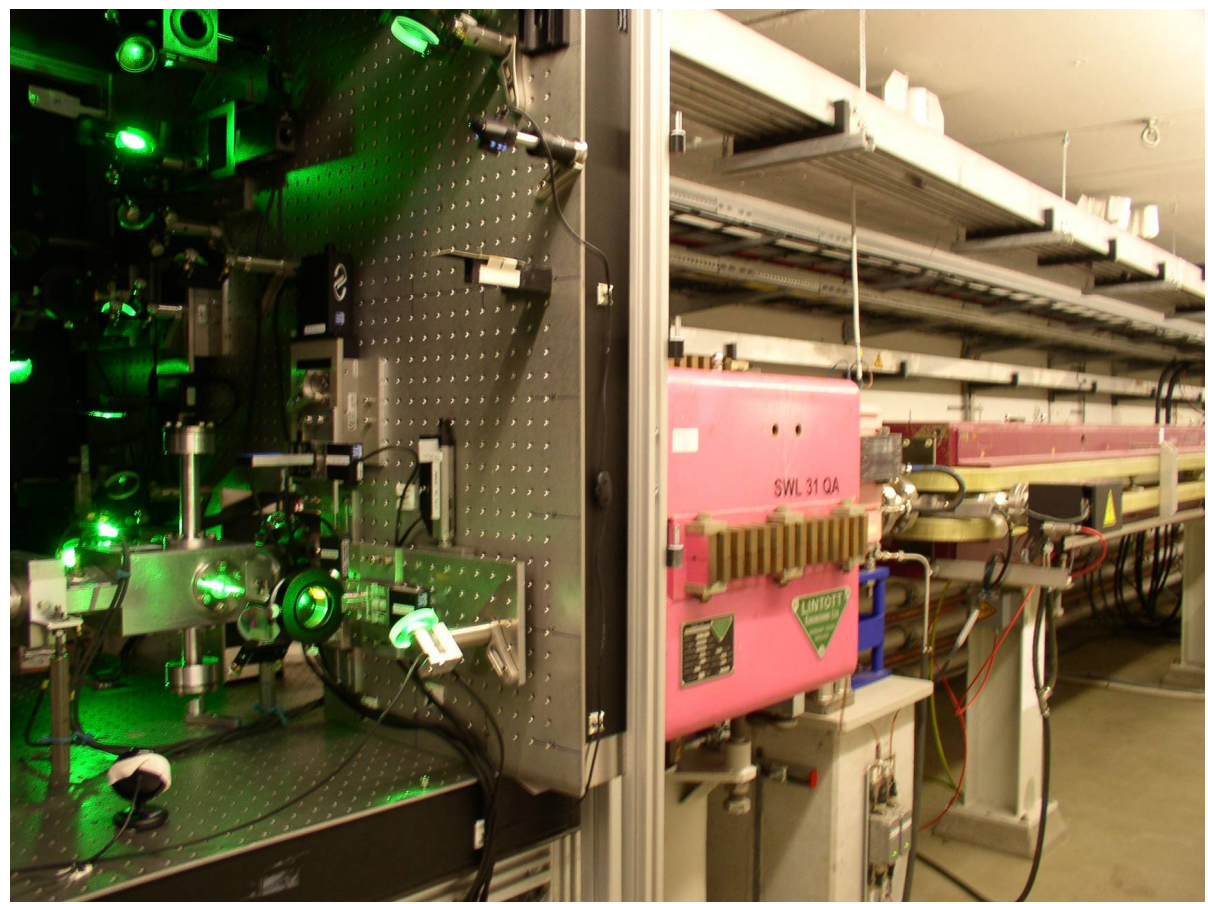

Figure 6. Existing beamline: interaction chamber surrounded by Laser-Wire optics with following quadrupole and dipole magnets. A green laser is shining through horizontal view port of the chamber. 
Table 3. Positions of beamline components.

\begin{tabular}{|c|c|c|c|c|c|}
\hline Component name & DB name & Position SWL (m) & Position vs IP (m) & \multirow{3}{*}{$\begin{array}{c}\gamma-e \text { beam separation } \\
X_{B}-X_{\gamma} \\
(\mathrm{cm})\end{array}$} & \multirow{3}{*}{$\begin{array}{c}e^{\prime}-e \text { beam separatior } \\
X_{e}-X_{B} \\
(\mathrm{~cm})\end{array}$} \\
\hline BPM-SL1 & BPM & 38.8295 & -6.5055 & & \\
\hline BPM-SL2 & BPM & 32.6000 & -0.2760 & & \\
\hline IP & LSW & 32.3240 & 0.0000 & 0.0000 & 0.0000 \\
\hline $\mathrm{Q}^{-}$ & Q5K & 31.3670 & 0.9570 & 0.0000 & 0.0000 \\
\hline Pickup & ВРM & 30.9365 & 1.3875 & 0.0000 & 0.0000 \\
\hline Dipole & DK & 27.4410 & 4.8830 & 4.8288 & 0.7745 \\
\hline $\mathrm{Q}^{+}$ & Q4K & 23.9980 & 8.3260 & 9.6576 & 1.5489 \\
\hline $\mathrm{Q}^{-}$ & Q2K & 13.9000 & 18.4240 & 37.9824 & 3.6515 \\
\hline $\mathrm{Q}^{+}$ & Q1K & 8.2000 & 24.1240 & 53.9708 & 8.2569 \\
\hline ВРМ- $X_{B}$ & BPM & 0.6000 & 31.724 & & \\
\hline e-window & - & 0.8240 & 31.8500 & 74.6604 & 14.2165 \\
\hline
\end{tabular}

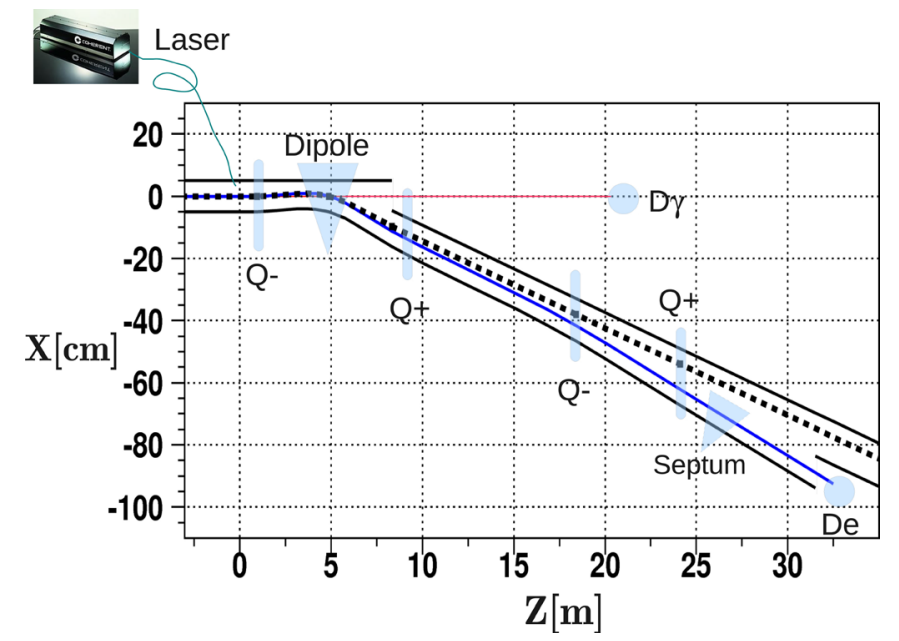

Figure 7. Beamline elements relative to laser and positron beam (dotted line) interaction point ( $Z=0 \mathrm{~m}, X=0 \mathrm{~cm}$ ). Beam pipe and exit windows are drawn in black, trajectories for Compton edge positrons and photons are shown in blue and red. $\mathrm{Q}(-)+$ are assigned to (de)focusing quadrupoles. Photon and electron detectors denoted by $D \gamma$ and De respectively.

(BPM-SL1, BPM-SL2) and bunch timing (Pickup) measurements [31]. Beam horizontal position evaluated by BPM- $X_{B}$ at SWL $0.6 \mathrm{~m}$ will enter space refractivity derivations.

Most important beamline element, apart the interaction chamber, is a $5.4 \mathrm{~m}$ dipole which will separate scattered positrons and photons from the PETRA (neutral) beam. Focusing and defocusing quadrupoles are assigned by Q+ and Q- respectively, relative to the horizontal (x) plane. Quadrupoles located downstream of the dipole will noticeably bend the Compton edge positrons because of considerable horizontal offsets from the quadrupole center. Such a bend is visible at the last defocusing quadrupole in Figure 7. Last focusing quadrupole bend should be compensated to achieve necessary separation between the extracted positron and neutral beam at the exit window. Characteristics of the dipole and quadrupole magnets [27] are displayed in Table 4 for the $6 \mathrm{GeV}$ machine and nominal beam optics conditions.

Separations between neutral beam and scattered photons and positrons are also included in Table 3 (last 2 columns). These distances are calculated at the geometrical centers of the magnets along z. Table shows sufficient outside (the ring) room to place a $\gamma$ detector starting about $25 \mathrm{~m}$ downstream of the interaction point while the Compton positrons could be comfortably detected at $31.5 \mathrm{~m}$, inside the ring.

Vacuum beam pipe downstream of the Q2K should be modified to allow extraction and detection of the Compton scattered positrons. For that the vacuum pipe has to be extended on inner side as it seen on Figure 7. The extension will end by a Titanium exit window of thickness $356 \mu \mathrm{m}$ and size $60 \mathrm{~mm} \times 20 \mathrm{~mm}$. In addition 
magnetic field of the last quadrupole Q1K should be shielded for scattered positrons. Otherwise focusing field of the quadrupole is considerably strong for off-center Compton edge positrons to bring them back to the beam. In case of technical difficulties for quadrupole field shielding, it is possible to use an additional dipole magnet. Right after the Q1K quadrupole there is sufficient separation (about $7 \mathrm{~cm}$ ) between the Compton positrons and neutral beam to accommodate a septum magnet.

In contrast to space birefringence, experiment for the refractivity requires absolute position measurements of the scattered Compton particles. Therefore, positioning and alignment of the $\gamma, e^{+}$detectors and the laser beam should be done with best available accuracy in horizontal plane. For absolute calibration of the BPM-XB a horizontal wire scanner should be installed in near vicinity of the BPM.

\section{Expected Performance}

\subsection{Compton Spectra}

For $6 \mathrm{GeV}$ PETRA and $355 \mathrm{~nm}$ laser, energy and angular distributions of the Compton positrons and photons are presented on Figure 8. Scattering kinematic factor is $x=0.16$ which corresponds to Compton edge positron

\begin{tabular}{|c|c|}
\hline \multicolumn{2}{|c|}{ Dipole } \\
\hline Length & $5.378 \mathrm{~m}$ \\
\hline Bending angle & $1.607^{\circ}$ \\
\hline Bending radius & $191.73 \mathrm{~m}$ \\
\hline Field & $0.10439 \mathrm{~T}$ \\
\hline Field Error $\quad \Delta B / B$ & $5 \times 10^{-4}$ \\
\hline Critical Energy & $2.499 \mathrm{keV}$ \\
\hline \multicolumn{2}{|c|}{ Quadrupoles } \\
\hline Length & $1042 \mathrm{~mm}$ \\
\hline Aperture & $50 \mathrm{~mm}$ \\
\hline Gradient (max) & $15 \mathrm{~T} / \mathrm{m}$ \\
\hline$k$ & $0.749 \mathrm{~m}^{-2}$ \\
\hline Field Error $\Delta k / k$ & $4 \times 10^{-3}$ \\
\hline
\end{tabular}

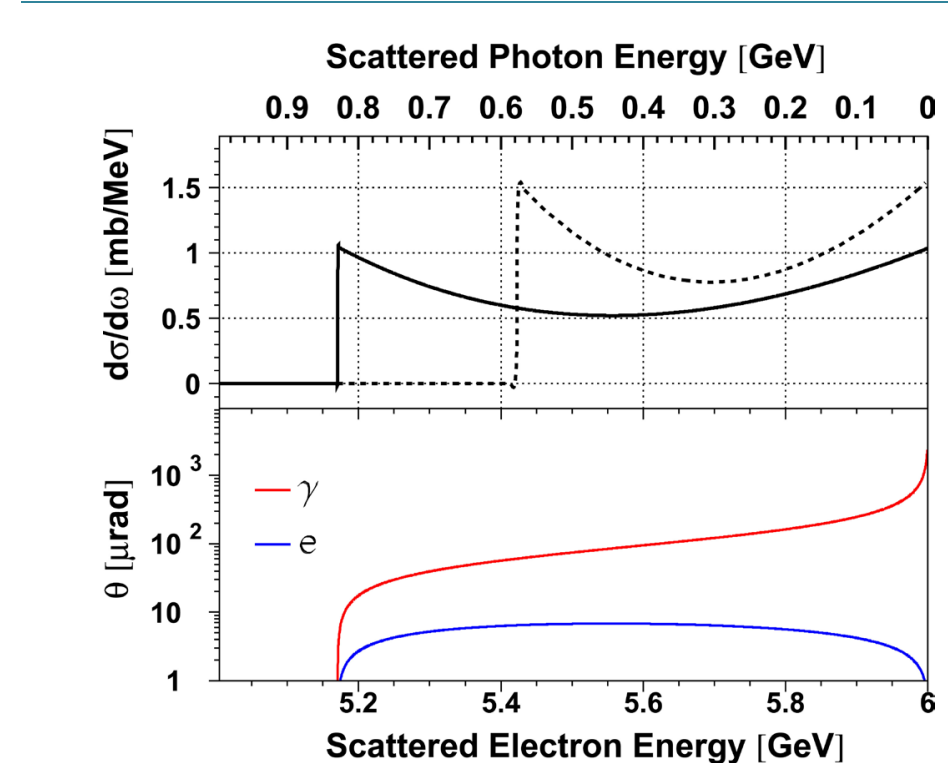

Figure 8. Compton cross-section and scattering angles for initial $6 \mathrm{GeV}$ positron and $3.49 \mathrm{eV}$ photon. Laser and $e^{+}$beam crossing angle is $90^{\circ}$. Dotted line spectrum is for $2.33 \mathrm{eV}$ green laser. 
(minimal) energy of $\mathcal{E}_{\min }^{\prime}=5.17 \mathrm{GeV}$. At this energy the scattered positron retains initial movement direction as it is clear from the plot displaying angular dependencies. Compton edge photons follow the same direction with an energy of $0.83 \mathrm{GeV}$.

Hunted gravitational effects will change energy sharing between the positron and photon. Expected changes are relatively small and will be hardly detectable by direct calorimetry so, we will use beamline magnets to convert scattered positron energy (momentum) to position in order to explore more sensitive instrumentation. To estimate spatial relationships we apply an approximate formula connecting energy and position of the scattered positron. Detected horizontal position of the scattered positron with energy $\mathcal{E}^{\prime}$ could be presented by

$$
X=X_{0}+\left(Z-Z_{D}\right)\left(\theta_{x}+\frac{e L B}{\mathcal{E}^{\prime}}\right)
$$

where $X_{0}, \theta_{x}$ are position and horizontal angle of the positron at the laser interaction point, $Z$ and $Z_{D}$ are locations of the detector and bending dipole respectively. Here $L$ and $B$ stand for the dipole length and magnetic field while influence of the quadrupoles is ignored. Equation (13) is derived from charged particle trajectory formula in a magnetic field [32] using high energy approximations $\mathcal{E}^{\prime} \gg m$ and $\theta_{x} \ll 1$. From this relation it follows that an energy change $\Delta \mathcal{E}^{\prime}$ around the Compton edge will produce a position change

$$
\Delta X=144.3 \Delta \mathcal{E}^{\prime} \mu \mathrm{m} / \mathrm{MeV}
$$

at the detector location $Z=32 \mathrm{~m}$.

\subsection{Smearing Factors}

Momentums and energies of Compton particles are smeared by initial laser and positron beam position, angular and energy distributions. We use Equation (13) with $e^{+}$beam optics parameters and numbers from Table 1, Table 2 to calculate magnitudes of smearing factors. Estimated influence of different factors on position of the Compton edge positron at detector location is shown in Table 5.

Apart from the mentioned main contributing factors Table 5 displays also smearing by multiple scattering in the positron exit window. Overall smearing is about $800 \mu \mathrm{m}$ which agrees to a detailed simulation results presented in Figure 9. Dominant smearing contributor is the lepton beam energy spread, quantifying as

$\sigma_{x \mathcal{E}} \approx \Delta X_{e e^{\prime}} \sigma_{\mathcal{E}} / \mathcal{E}$ with $\Delta X_{e e^{\prime}}$ being separation between scattered positron and neutral beam at detector location. Hence, one can reduce smearing induced by inter-bunch energy spread only via moving detector closer to IP with an expense of shrinking available place for the $e^{\prime}$ detectors.

\subsection{Rates}

Compton secondaries will be detected in single particle resolving regime with about 0.01 particles per bunch. PETRA mostly operates with 40, 60 or 240 bunches [34] with corresponding inter-bunch spacings of $192 \mathrm{ns,}$ $128 \mathrm{~ns}$ and 32 ns. Therefore, expected Compton rates are $52 \mathrm{kHz}, 78 \mathrm{kHz}$ and $313 \mathrm{kHz}$ for different bunch modes of the machine. However, since we are interested exceptionally on the Compton edge particles, the rates can further be reduced by discriminating energies of the photons or positrons. Assuming $5 \%$ energy detection resolution for single particles the rates could be reduced by a factor of 3 or 12 triggering on the positron or photon calorimeter respectively. This numbers are derived by integrating spectrum on Figure 8 within ranges of $0 \%$ to $100 \%$ and $95 \%$ to $100 \%$.

\subsection{Detectors}

In previous sections we have defined energy and rate of the expected signals. For $e^{+}$and $\gamma$ produced at Compton

\begin{tabular}{ccc} 
Table 5. Smearing factors. & \\
\hline Factor & Value & $\mathcal{E}^{\prime}$ smearing \\
\hline$e^{+}$-laser IP & $\sigma_{I P \times}=10 \mu \mathrm{m}$ & $10 \mu \mathrm{m}$ \\
$e^{+}$energy spread & $\sigma_{\mathcal{E}} / \mathcal{E}=10^{-3}$ & $750 \mu \mathrm{m}$ \\
$e^{+}$divergence & $\sigma_{x^{\prime}}=10 \mu \mathrm{rad}$ & $280 \mu \mathrm{m}$ \\
e-window & $\sigma_{\text {Mult }}=2.4 \mathrm{mrad}$ & $50 \mu \mathrm{m}$ \\
\hline
\end{tabular}


edge, simultaneous position and energy measurements are necessary. We intend to install a combination of position sensitive and calorimetric detectors at the positron and photon branch. For energy measurements homogeneous crystal calorimeters could provide a resolution of $5 \%$ over $\sqrt{\mathcal{E}}$. Position measurements will be performed with silicon strip or pixel detectors. A position resolution of $10 \mu \mathrm{m}$ and rate capability of $30 \mathrm{kHz}$ would be sufficient for the whole range of our measurements. The silicon detector will be placed in front of calorimeter for positrons while for the photons position detector will be located at middle part of the calorimeter where shower lateral size is maximal. This arrangement is displayed in Figure 10.

\subsection{Backgrounds}

Apart from the laser Compton scattering there are other beam related sources of scattered photons or leptons at any accelerator environment. These particles may enter detectors and spoil measured distributions. For storage rings one should first consider synchrotron radiation from bending or quadrupole magnets [32]. In our case positron detectors are located inside the ring and could see only scattered synchrotron light while the ouside photon detectors are imposed to direct synchrotron radiation. Therefore we plan to shield detectors at the beam pipe side and, in addition, the photon detectors also at the front side to absorb completely the synchrotron radiation.

Other potentially dangerous processes are beam-gas interaction [35], scattered blackbody radiation [36] [37] and intra-bunch scattered (Touschek) positrons [38]. Explored positron and photon coincidence registration

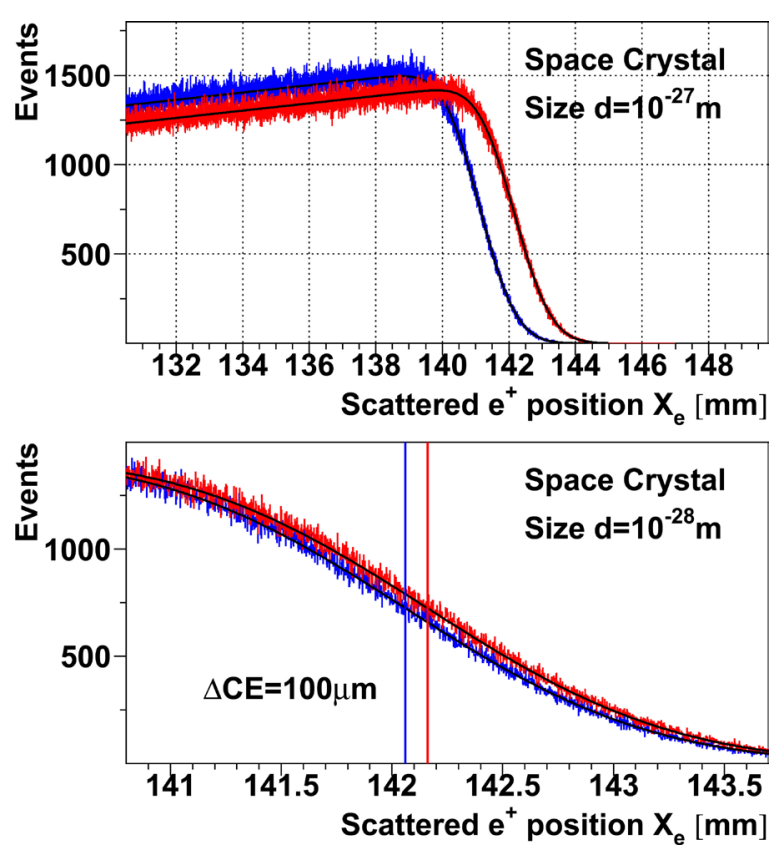

Figure 9. Simulation results: scattered positron spatial spectra for laser left and right helicities. Horizontal scale is distance between the scattered $e^{+}$and neutral beam at the detector location. Lower plot shows Compton Edge (CE) positions obtained by fitting a 4 parameter function from Ref. [33] to spectra. Same function is applied for fitting upper plot distributions.

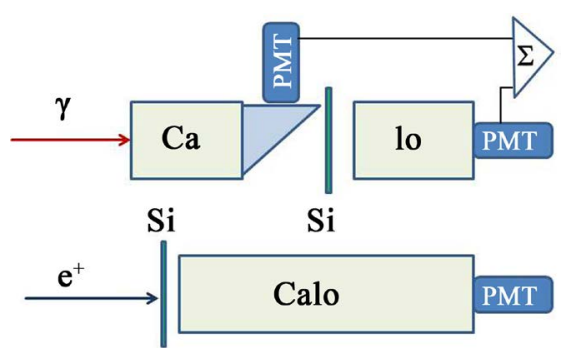

Figure 10. Photon and positron detectors schematics. Position sensitive part is denoted by "Si” and energy sensitive part by “Calo”. PMT stands for photomultiplier. 
mode will greatly suppress or completely eliminate backgrounds from the thermal photons and Touschek positrons. The beam-gas Bremsstrahlung, however, can not be discriminated since the energy balance is similar to the Compton scattering. This would be the main background and it should be handled by keeping vacuum pressure around the Compton interaction point possibly low (mounting of additional vacuum pumps should be foreseen). The Bremsstrahlung rate will be monitored periodically by blocking the laser light by a shutter. Alternatively a fast, electro-optical or acousto-optical modulator may be used to redirect the laser out of certain portion of bunches for background measurements.

A more severe source of background at the SW section could be aperture limitations-beam collimators (coll 1 and coll 2) of PETRA are located about $15 \mathrm{~m}$ upstream and downstream of our detectors.

\subsection{Measurements}

\subsubsection{Space Birefringence}

As it was described above for space chirality measurements the laser helicity will be flipped with few hundred Hertz sweeping frequency to avoid correlations with any possible periodic source. Accumulated spatial events will be tagged with the helicity and the resulted spectra (simulated samples are shown on Figure 9) will be analyzed to fetch the Compton edge for each helicity. For that the spectra could be fitted by Gaussian and error combined functions as it is proposed in [33] or by Compton cross-section convoluted with detector resolution, as in [20]. An example of fitted spectra is shown in Figure 9, where a Compton edge shift of $100 \mu \mathrm{m}$ is detected by fits for simulated initial space birefringence at $\xi=10^{7}\left(\approx 10^{-28} \mathrm{~m}\right)$. After the fits an asymmetry

$$
A=\frac{X_{e}^{+}-X_{e}^{-}}{X_{e}^{+}+X_{e}^{-}}
$$

will be calculated with $X_{e}^{+}, X_{e}^{-}$being Compton edge positions for positive and negative helicities. Finally, this measured asymmetry will be related to Equation (11).

\subsubsection{Space Refractivity}

For vacuum index measurement it is necessary to detect absolute energy of the Compton edge positron or photon. This could be accomplished by simultaneous measurements of the photon $X_{\gamma}$, positron $X_{e}$, and neutral beam $X_{B}$ positions $^{1}$ (see Figure 3). With this information one can use Equation (8) and Equation (13) to arrive to

$$
n-1=\frac{1}{2 \gamma^{2}}\left(x \frac{\left(X_{B}-X_{\gamma}\right)^{2}}{\left(X_{e}-X_{B}\right)\left(X_{e}-X_{\gamma}\right)}-1\right)
$$

which holds if the scattered positron and the neutral beam are transported through the same (strength) magnetic field, i.e. for homogeneous dipole field. With quadrupoles one needs to apply corrections which could be measured during calibrating quadrupole scans. This is, still, not the full story, since any small offset in beam energy value $\mathcal{E}_{\text {beam }}$ would result in a fake refractivity measurement. Therefore, for space refractivity experiment it is necessary an independent, precise measurement of the beam absolute energy. Moreover, since the beam energy changes along the ring, the energy measurement should be done at the Compton interaction point. For this we will explore a different frequency light generated by the same or another laser. Since the UV $355 \mathrm{~nm}$ light is third harmonic of the $1064 \mathrm{~nm}$ Nd:YAG laser, we can use second harmonic, $532 \mathrm{~nm}$ wavelength which otherwise is widely available as a standalone laser solution.

Combining Equation (8), Equation (7) and Equation (13) for two laser photon energies, after some lengthy though simple calculations we obtain expressions for beam energy and refractive space size measurements

$$
\begin{gathered}
\mathcal{E}_{\text {beam }}[\mathrm{GeV}]=\frac{u_{1}+u_{2} L_{2}+u_{3} L_{1}+u_{4} L_{1} L_{2}}{u_{5}+u_{6} L_{2}+u_{7} L_{1}+u_{8} L_{1} L_{2}} \\
\zeta l_{P}[\mathrm{~m}]=7 \times 10^{-24} \frac{v_{1}+v_{2} L_{2}+v_{3} L_{1}+v_{4} L_{1} L_{2}+v_{5} L_{1}^{2}+v_{6} L_{1}^{2} L_{2}}{v_{7}+v_{8} L_{2}+v_{9} L_{1}+v_{10} L_{1} L_{2}+v_{11} L_{1}^{2}+v_{12} L_{1}^{2} L_{2}}
\end{gathered}
$$

${ }^{1}$ Method is similar to a 3-positions measurement scheme proposed for ILC energy determination [39]. 
where $L_{1}, L_{2}$ are incorporating position measurements for the laser 1 (UV, $3.49 \mathrm{eV}$ ) and laser 2 (green, $2.33 \mathrm{eV}$ ) and

$$
L=\frac{X_{e}-X_{B}}{X_{B}-X_{\gamma}}
$$

is a common expression to calculate $L_{1}, L_{2}$. Coefficients $u$ and $v$ depend solely on two laser wavelengths, crossing angles and a central energy of $e^{+}$beam chosen to be $\mathcal{E}_{0}=6.00000 \mathrm{GeV}$. Resulting expressions are too long to be presented here, hence we display numerical values of the coefficients in Table 6 .

Described formalism allows to measure space refractivity provided UV and green lasers to be delivered to the same interaction point. Spatial separation of Compton edge positrons from two lasers will be $40.915 \mathrm{~mm}$ thus allowing to use the same exit window and $e^{+}$detector.

\subsubsection{Space Anisotropy}

Possible spatial dependence of space birefringence or refractivity will be tested by writing Equation (12) for PETRA declination angle $\delta=22.58^{\circ}$ at interaction point

$$
Q=Q_{0}\left(0.92 \cos \delta_{0} \cos \left(\alpha-\alpha_{0}\right)+0.38 \sin \delta_{0}\right)
$$

with $Q=\Delta n$ for measured birefringence or $Q=n-1$ for refractivity. For each measurement time a corresponding right ascension angle $\alpha$ will be calculated and obtained $Q-\alpha$ dependence will be fitted by Equation (19) to find space anisotropy axis direction $\delta_{0}, \alpha_{0}$.

\section{Experimental Reach and Accuracy}

\subsection{Statistical Errors}

For an expected small asymmetry from Equation (14) $X_{e}^{+} \approx X_{e}^{-}=X_{e} \approx 142 \mathrm{~mm}$, error propagation gives

$$
\Delta A=\frac{1}{\sqrt{2}} \frac{\Delta X_{e}}{X_{e}}
$$

where $\Delta X_{e}$ is accuracy of the Compton edge ( $X_{e}$ ) measurement. Although Compton edge is derived by fitting distribution with many events, for statistical error estimation it is more convenient evaluating a single event accuracy which allows a direct application of conventional statistical events-strength formalism. Thus, we can assign $\sigma_{X_{e}} \approx 800 \mu \mathrm{m}$ as position error for a single event equal to the position smearing derived above, and get $\Delta X_{e}=\sigma_{X_{e}} / \sqrt{T R_{e}}$, where $R_{e}$ is rate of $e^{+}$events around Compton edge and $T$ is time of measurement. Necessary data taking times to achieve different sensitivities, for an average rate of $R_{e}=13 \mathrm{kHz}$ (estimated from 2011 running [34]), are displayed on Table 7.

On a way to calculate refractivity measurement errors we estimate spreads of Equation (18) constituents. A Compton photon position at detector location, $X_{\gamma}$, defines initial angle $\theta_{x}$. Hence, the difference $X_{e}-X_{\gamma}$ will be free from fluctuations of the $\theta_{x}$. The beam position $X_{B}$ is a measure of magnetic field strength which

Table 6. Coefficients in Equation (16) and Equation (17).

\begin{tabular}{cc}
\hline$u_{1}=0.03747513690$ & $u_{2}=-8.245513915$ \\
$u_{3}=3.281272436$ & $u_{4}=-5.001716619$ \\
$u_{5}=-0.04417775880$ & $u_{6}=0.8269285477$ \\
$u_{7}=-0.7135896078$ & $u_{8}=0.1575166987$ \\
$v_{1}=0.00100246550$ & $v_{2}=-0.2205687272$ \\
$v_{3}=0.1319522859$ & $v_{4}=-0.9607252134$ \\
$v_{5}=0.7135896078$ & $v_{6}=-0.1575166987$ \\
$v_{7}=-0.04417775880$ & $v_{8}=0.8269285477$ \\
$v_{9}=-0.7577673666$ & $v_{10}=0.9844452464$ \\
$v_{11}=-0.7135896078$ & $v_{12}=0.1575166987$ \\
\hline
\end{tabular}


Table 7. Asymmetry measurement times and space birefringence sensitivities.

\begin{tabular}{ccc}
\hline$\Delta A$ & $\xi l_{p}$ & $T$ \\
\hline $10^{-5}$ & $10^{-28} \mathrm{~m}$ & $12 \mathrm{sec}$ \\
$10^{-6}$ & $10^{-29} \mathrm{~m}$ & $20 \mathrm{~min}$ \\
$10^{-7}$ & $10^{-30} \mathrm{~m}$ & 34 hours \\
$10^{-8}$ & $10^{-31} \mathrm{~m}$ & 141 days \\
\hline
\end{tabular}

is completely defined if we explore beam direction (slope) at the interaction point, measured by two upstream BPMs. From BPM resolution of $5 \mu \mathrm{m}$ per bunch traverse [40] we arrive to following accuracy estimators for a Compton scattering event:

$$
\begin{aligned}
& \sigma\left(X_{\gamma}\right)=20 \mu \mathrm{m} \\
& \sigma\left(X_{B}\right)=15 \mu \mathrm{m} \\
& \sigma\left(X_{e}\right)=752 \mu \mathrm{m}
\end{aligned}
$$

the latter is a quadratic sum of smearings by beam energy and exit window from Table 5. Error propagation applied to Equation (17), with derived numbers, yields an accuracy of $5 \times 10^{-26} \mathrm{~m}$ for $\zeta l_{P}$ from two (UV and green) Compton edge scattering events. Evaluating Equation (16) with the same events we find a $300 \mathrm{MeV}$ statistical error for $\mathcal{E}_{\text {beam }}$. At PETRA this would apply $50 \mathrm{sec}$ data taking time at $5 \mathrm{kHz}$ for beam energy measurement with a $10^{-3}$ relative statistical error. A sensitivity of $10^{-28} \mathrm{~m}$ for space refractivity will be achieved during the same time period.

\subsection{Systematic Effects}

\subsubsection{Space Birefringence}

In general, it is a difficult task to mention asymmetry limiting sources a priori since most (theoretically all) of beam and detector parameters and their drifts are not (should not be) affected by helicity flips and are ignorable for asymmetries. Therefore, we refer to asymmetry measurement achievements of former accelerator experiments. Asymmetries as small as $10^{-7}$ have been detected with a sensitivity $10^{-8}$ at the SLAC $50 \mathrm{GeV}$ experiments [41] [42], based on beam helicity flips. Same order sensitivities for measured asymmetries are reported at the MAMI $1 \mathrm{GeV}$ experiments [43] [44]. Thus, similar accuracies seem reachable at PETRA which suggests that the $6 \mathrm{GeV}$ machine could test space birefringence down to $10^{-31} \mathrm{~m}$.

There are few potential sources of false asymmetry which are correlated with laser helicity flips and are all related to either the laser or lepton beam polarization. These are positron beam longitudinal or transverse polarization and laser light linear polarization. Introduced asymmetries by the mentioned factors, for $100 \%$ polarization, are plotted on Figure 11. First we note that none of the quoted factors could shift the Compton edge, although, intensity changes displayed on Figure 11, convoluted with detectors responses, could mimic a shift of the edge. However, positron beam longitudinal polarization in PETRA should be plain zero-otherwise the proposed setup is able to measure and monitor even small amounts of it. Situation is different for the circular laser light where always a small fraction of linear component exist [45] and for the PETRA beam transverse polarization which could be acquired by Sokolov-Ternov mechanism [46]. Nevertheless, since intensity changes produced by helicity flips are vanishing at the Compton edge, contribution of these two factors to gravitation induced asymmetry should be negligible.

\subsubsection{Space Refractivity}

Limiting factor for refractivity measurement is positron beam energy uncertainty $\Delta \mathcal{E}$. We can calculate corresponding effect on refractivity measurement using Equation (8) by explicitly writing $\gamma$ and $x$ dependence on $\mathcal{E}$ at the Compton edge. Resulting expression

$$
\Delta(n-1)=\frac{1}{2 \gamma^{2}} \frac{\Delta \mathcal{E}}{\mathcal{E}}
$$

sets an accuracy limit for refractivity measurement with the proposed method. With $\Delta \mathcal{E} / \mathcal{E} \approx 10^{-3}$ a systematic 


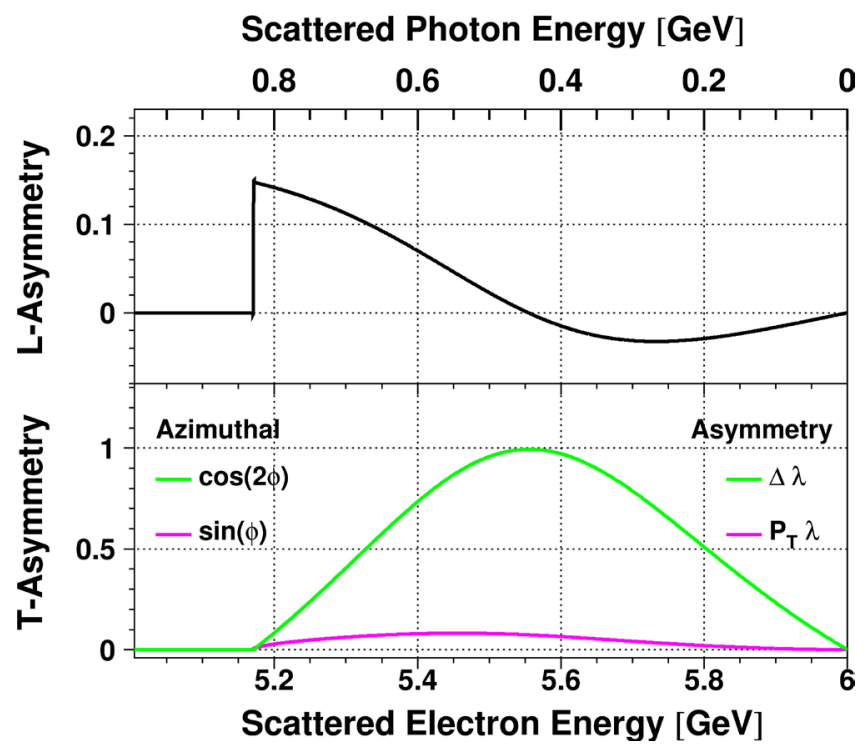

Figure 11. Compton cross-section asymmetry produced by laser helicity flip. Upper plot: for longitudinal positrons. Lower plot: for transversal (magenta) or unpolarized (green) positrons. In latter case laser polarization is linear.

Table 8. Equipment expenses.

\begin{tabular}{cc}
\hline Component & Cost $(€)$ \\
\hline Laser system & 250,000 \\
Beam pipe & 75,000 \\
Septum magnet & 75,000 \\
Detectors & 150,000 \\
Total & 550,000 \\
\hline
\end{tabular}

error for refractivity is $3.6 \times 10^{-12}$ which corresponds to $\zeta=7.3 \times 10^{6}$ and an experimental reach to space crystal size of $1.2 \times 10^{-28} \mathrm{~m}$. Bending field inhomogeneity should contribute twice as less as energy spread since it enters to Equation (13) together with the energy and has $5 \times 10^{-4}$ relative uncertainty.

\section{Cost Estimate}

Since most of hardware should be adapted to existing beamline and magnets, there are no standard components available and therefore, we can only roughly estimate amount of necessary expenses. Our estimates are shown in Table 8.

Prices for optics and electronics are included in laser and detector costs respectively.

\section{Conclusions}

A simple theoretical framework is established allowing to access extremely small distances in laboratory, provided a vacuum refraction index growing with photon energy. Such vacuum is suggested by wide range of gravity theories which predict space-time modifications around Planck scale. Motivated by these predictions, we propose a laser Compton experiment at PETRA to test empty space for single or double refraction. Experiment would be able to prove or reject crystal-space hypothesis reaching distances as small as $10^{-28} \mathrm{~m}$ for refractivity and $10^{-31} \mathrm{~m}$ for birefringence. Space isotropy measurements within these magnitudes are also foreseen.

Space birefringence measurements would be performed with UV polarized laser and would require 282 days of data taking (50\% efficiency assumed) to reach $10^{-31} \mathrm{~m}$ sensitivity. Probing space isotropy within this running period is possible with a sensitivity $10^{-30} \mathrm{~m}$ by mapping $360^{\circ}$ celestial circle with $3.6^{\circ}$ steps.

For space refractivity tests one should explore an additional green laser which will enable beam energy precise determination. Very fast, sub-minute measurement times are sufficient to sample refractivity with an accu- 
racy which corresponds to $10^{-28} \mathrm{~m}$ distance sensitivity.

Observation of either refractive or birefringent Planck space will have a large impact on gravity and related fields.

\section{Acknowledgements}

We are grateful to Reinhard Brinkmann for his support and advices related to the beam setup. One of the authors (V.G.) thanks Stefan Schmitt and Daniel Pitzl for useful discussions.

\section{References}

[1] Amsler, C., et al. (2008) Physics Letters B, 667, 1. http://dx.doi.org/10.1016/j.physletb.2008.07.018

[2] Amelino-Camelia, G., Ellis, J.R., Mavromatos, N.E., Nanopoulos, D.V. and Sarkar, S. (1998) Nature, $393,763$. http://dx.doi.org/10.1038/31647

[3] Gambini, R. and Pullin, J. (1999) Physical Review D, 59, Article ID: 124021.

[4] Gharibyan, V. (2012) Physical Review Letters, 109, Article ID: 141103. http://dx.doi.org/10.1103/PhysRevLett.109.141103

[5] Amelino-Camelia, G. (2013) Living Reviews in Relativity, 16, 5. http://dx.doi.org/10.12942/lrr-2013-5

[6] Mavromatos, N.E. (2009) Journal of Physics: Conference Series, 171, Article ID: 012007. http://dx.doi.org/10.1088/1742-6596/171/1/012007

[7] Gleiser, R.J., Kozameh, C.N. and Parisi, F. (2003) Classical and Quantum Gravity, 20, 4375. http://dx.doi.org/10.1088/0264-9381/20/20/303

[8] Gubitosi, G., Genovese, G., Amelino-Camelia, G. and Melchiorri, A. (2010) Physical Review D, 82, Article ID: 024013. http://dx.doi.org/10.1103/PhysRevD.82.024013

[9] Hehl, F.W., Von Der Heyde, P., Kerlick, G.D. and Nester, J.M. (1976) Reviews of Modern Physics, $48,393$. http://dx.doi.org/10.1103/RevModPhys.48.393

[10] Prasanna, A.R. and Mohanty, S. (2009) General Relativity and Gravitation, 41, 1905. http://dx.doi.org/10.1007/s10714-009-0790-1

[11] Myers, R.C. and Pospelov, M. (2003) Physical Review Letters, 90, Article ID: 211601. http://dx.doi.org/10.1103/PhysRevLett.90.211601

[12] Dalvit, D.A.R., Mazzitelli, F.D. and Molina-Paris, C. (2001) Physical Review D, 63, Article ID: 084023. http://dx.doi.org/10.1103/PhysRevD.63.084023

[13] Ellis, J.R., Mavromatos, N.E. and Nanopoulos, D.V. (2008) Physics Letters B, 665, 412. http://dx.doi.org/10.1016/j.physletb.2008.06.029

[14] Dittrich, W. and Gies, H. (1998) Physical Review D, 58, Article ID: 025004. http://dx.doi.org/10.1103/PhysRevD.58.025004

[15] Bombelli, L. and Winkler, O. (2004) Classical and Quantum Gravity, 21, L89. http://dx.doi.org/10.1088/0264-9381/21/12/L01

[16] Gleiser, R.J. and Kozameh, C.N. (2001) Physical Review D, 64, Article ID: 083007. http://dx.doi.org/10.1103/PhysRevD.64.083007

[17] Stecker, F.W. (2011) Astroparticle Physics, 35, 95-97. http://dx.doi.org/10.1016/j.astropartphys.2011.06.007

[18] McGlynn, S., Clark, D.J., Dean, A.J., Hanlon, L., McBreen, S., Willis, D.R., McBreen, B., Bird, A.J. and Foley, S. (2007) Astronomy \& Astrophysics, 466, 895-904. http://dx.doi.org/10.1051/0004-6361:20066179

[19] Heyman, D., Hinteleitner, F. and Major, S. (2004) Physical Review D, 69, Article ID: 105016. http://dx.doi.org/10.1103/PhysRevD.69.105016

[20] Gharibyan, V. (2005) Physics Letters B, 611, 231-238. http://dx.doi.org/10.1016/j.physletb.2005.02.053

[21] Ellis, J.R., Mavromatos, N.E. and Nanopoulos, D.V. (2009) Physics Letters B, 674, 83-86. http://dx.doi.org/10.1016/j.physletb.2009.02.030

[22] Lipps, F.W. and Tolhoek, H.A. (1954) Physica, 20, 385-405.

[23] McMaster, W.H. (1961) Reviews of Modern Physics, 33, 8-28. http://dx.doi.org/10.1103/RevModPhys.33.8

[24] Sudarsky, D., Urrutia, L. and Vucetich, H. (2002) Physical Review Letters, 89, Article ID: 231301. http://dx.doi.org/10.1103/PhysRevLett.89.231301 
[25] Marx, M., Chrin, J., Martin, I., Muekaphael and Schaa, V.R.W., Eds. (2011) Beam Diagnostics and Instrumentation for Particle Accelerators. 10th European Workshop on Beam Diagnostics and Instrumentation for Particle Accelerators, Hamburg, 16-18 May 2011, TUPD21, 350.

[26] Balewski, K., Brefeld, W., Decking, W., Franz, H., Rohlsberger, R. and Weckert, E., Eds. (2004) PETRA III: A Low Emittance Synchrotron Radiation Source. Technical Design Report, DESY-04-035.

[27] Machine Parameters PETRA III (Design Values). http://hasylab.desy.de/facilities/petra_iii/machine/parameters/

[28] Accelerators at DESY, PETRA III. http://m.desy.de/accelerators/

[29] Paladin Advanced 355, Modelocked UV Laser. http://www.coherent.com/downloads/PaladinAdvanced355_DSrevB_0112_2.pdf

[30] Aumeyr, T., Blair, G.A., Boogert, S., Boorman, G., Bosco, A., Balewski, K., Elsen, E., Gharibyan, V., et al. (2010) A 2-D Laser-Wire Scanner at PETRA-III. 1st International Particle Accelerator Conference, Tokyo, 23-28 May 2010, MOPE069, 1137-1139.

[31] Chrin, J., Marx, M., Schaa, V.R.W. and Schlott, V., Eds. (2009) Beam Diagnostics and Instrumentation for Particle Accelerators. 9th European Workshop on Beam Diagnostics and Instrumentation for Particle Accelerators, Basel, 25-27 May 2009, MOPD20, 92.

[32] Chao, A.W. and Tigner, M., Eds. (2006) Handbook of Accelerator Physics and Engineering. World Scientific Publishing, Singapore, $702 \mathrm{p}$.

[33] Klein, R., Thornagel, R., Ulm, G., Mayer, T. and Kuske, P. (1997) Nuclear Instruments and Methods in Physics Research Section A: Accelerators, Spectrometers, Detectors and Associated Equipment, 384, 293-298. http://dx.doi.org/10.1016/S0168-9002(96)00899-6

[34] Alexander, G. and Ilka, F., Eds. (2012) Accelerators 2011: Highlights and Annual Report. DESY, Hamburg, 87 p.

[35] Le Duff, J. (1985) Nuclear Instruments and Methods in Physics Research Section A: Accelerators, Spectrometers, Detectors and Associated Equipment, 239, 83-101. http://dx.doi.org/10.1016/0168-9002(85)90702-8

[36] Telnov, V.I. (1987) Nuclear Instruments and Methods in Physics Research Section A: Accelerators, Spectrometers, Detectors and Associated Equipment, 260, 304-308. http://dx.doi.org/10.1016/0168-9002(87)90093-3

[37] Dehning, B., Melissinos, A.C., Perrone, F., Rizzo, C. and von Holtey, G. (1990) Physics Letters B, 249, 145-148. http://dx.doi.org/10.1016/0370-2693(90)90540-M

[38] Bernardini, C., Corazza, G.F., Di Giugno, G., Ghigo, G., Querzoli, R., Haissinski, J., Marin, P. and Touschek, B. (1963) Physical Review Letters, 10, 407-409. http://dx.doi.org/10.1103/PhysRevLett.10.407

[39] Muchnoi, N., Schreiber, H.J. and Viti, M. (2009) Nuclear Instruments and Methods in Physics Research Section A: Accelerators, Spectrometers, Detectors and Associated Equipment, 607, 340-366.

[40] Kube, G., Balewski, K., Brenger, A., Duhme, H.T., Gharibyan, V., Klute, J., Knaack, K., Krouptchenkov, I., et al. (2008) Overview of the Diagnostics Systems of PETRA III. 11th Biennial European Particle Accelerator Conference, Genoa, 23-27 June 2008, TUPC111, 1323-1325.

[41] Anthony, P.L., et al. and SLAC E158 Collaboration (2004) Physical Review Letters, 92, Article ID: 181602. http://dx.doi.org/10.1103/PhysRevLett.92.181602

[42] Anthony, P.L., et al. and SLAC E158 Collaboration (2005) Physical Review Letters, 95, Article ID: 081601. http://dx.doi.org/10.1103/PhysRevLett.95.081601

[43] Maas, F.E. and the A4-Collaboration (2005) The European Physical Journal A-Hadrons and Nuclei, 24, 47-50.

[44] Maas, F.E. and the A4-Collaboration (2008) AIP Conference Proceedings, 1056, 98-105. http://dx.doi.org/10.1063/1.3013086

[45] Barber, D.P., Bremer, H.D., Boge, M., Brinkmann, R., Bruckner, W., Buscher, C., Chapman, M., Coulter, K., et al. (1993) Nuclear Instruments and Methods in Physics Research Section A: Accelerators, Spectrometers, Detectors and Associated Equipment, 329, 79-111. http://dx.doi.org/10.1016/0168-9002(93)90924-7

[46] Sokolov, A.A. and Ternov, I.M. (1964) Soviet Physics—Doklady, 8, 1203-1205. 